\title{
Rad51 stabilizes the ends of two adjacent DSBs in yeast
}

\author{
Techang Chen ${ }^{1}$, Xiaoqi $\mathrm{Li}^{1}$, Ning Ma ${ }^{1}$, Jingbo Shan ${ }^{1}$, Peiyan $\mathrm{Guo}^{1}$, Sa Zhou ${ }^{1, \mathrm{a}}$ and Wenjian Ma ${ }^{1, \mathrm{a}}$ \\ ${ }^{1}$ Key Laboratory of Industrial Microbiology, Ministry of Education and Tianjin City, College of Biotechnology, Tianjin University of \\ Science and Technology
}

\begin{abstract}
DNA double-strand breaks (DSBs) are a major form of DNA damage, and its accurate repair is critical for maintaining genomic stability and preventing cancer. Repair of DSBs is mainly through two pathways, homology-directed DNA repair (HDR) and non-homologous end joining (NHEJ). Compared with NHEJ, HDR has higher fidelity, so the study of interrelated factors in homologous recombination pathway is of particularly important. In this paper, we constructed an I-SceI model controlled by a galactose promoter that produces a DSB or two adjacent DSBs on a single chromosome. Rad50 and Rad51 genes are further deleted in these models. Sensitivity experiments show that Rad51 stabilizes the ends of two adjacent DSBs in yeast. Deletion of Rad51 gene causes the sequence between two adjacent DSBs to drop out directly to form a large gap. If this gap is larger, the efficiency of NHEJ will be greatly reduced, resulting in the deaths of the strain. Our research shows that Rad51 stabilizes the ends of two adjacent DSBs in yeast.
\end{abstract}

\section{Introduction}

Rad51 protein is the first homology-directed DNA repair (HDR) associated protein found in mammalian cells and is the most critical enzyme for catalyzing HDR in vivo [1]. Rad51 can promote the binding of DNA strands and the multimerization of DNA ends, making nuclear protein filaments complementary to DNA gaps, mediating their transfer and annealing. Rad51 is involved in a complex network that includes DNA damage, cell cycle, apoptosis regulation, gene mutations, and protein abnormalities [2].

Overexpression of Rad51 can alter the recombination pathway, causing chromosome rearrangement, leading to abnormal karyotype expression, which is closely linked to its carcinogenicity. The functional study of $\operatorname{Rad} 51$ helps to strengthen the chemoresistance of tumors. The current study found that mice with deleted the Rad51 gene showed growth arrest in the embryonic stage and a large number of broken chromosomes in the cell [3]. Rad51 is highly expressed in many human tumors, including cervical cancer [4], Non-small cell lung cancer [5], breast cancer [6], ovarian cancer [7], pancreatic cancer [8] and so on. Rad51-related changes in DNA, mRNA, and protein levels may affect the development and progression of various tumors. This is explained by the fact that the $\operatorname{Rad} 51$ protein is a key protein in homologous recombination (HR), and $\mathrm{HR}$ is an important part of repairing DNA damage. For the time being, there continue to be many questions about the complex regulatory mechanism of rad51, which needs further research.

Here, we used the I-SceI homing endonuclease to cleave the DNA double strands and constructed three strains of I-SceI controlled by the galactose promoter, which produced two adjacent DSBs on a single chromosome induced by galactose. Compared to a single DSB, two adjacent DSB repairs require Rad51. If rad51 is deleted, the two adjacent DSB intermediate sequences will fall, resulting in a large gap, resulting in the death of the strain. This discovery is conducive to our further understanding of the rad51 functional mechanism, rad51 has the function of stabilizing the ends of the two DSBs to prevent the intermediate sequence from falling.

\section{Materials and Methods}

\subsection{Yeast Strains}

The original strain used in this experiment was derived from strain mwj49. (MAT $\alpha$ leu2-3,112 ade5-1 his7-2 ura3 $\Delta$ trp1-289), which contain a circular form of chromosome III (Chr III) [9]. The screening markers used is G418 (kanMX4), hy-gromycin (hphMX4) or nourseothricin (natMX4) [10]. Gal-I-SceI cassette amplified from plasmid pGSHU, insert Gal-I-SceI cassette in Chr III 91024-91143, between SUP53 and LEU2. Successfully constructed strains that did not produce DSB (no-DSBu), produced a DSB (one-DSBu ) and produced two adjacent DSBs (two-DSBu). The deletion mutants rad50, rad51, and their derived double or triple mutants were created by replacing the relevant ORF in these strains with selectable markers as previously described.

\subsection{Yeast sensitivity test}

Adequate yeast cells were transferred to a $10 \mathrm{ml}$ YPDA liquid medium in a shake flask the night before, and

${ }^{a}$ Corresponding author:ma_wj@tust.edu.cn, zhousa@tust.edu.cn 
cultured overnight at $30^{\circ} \mathrm{C}$ shaker. After the next day of Nocodazole treatment for 3 hours (synchronize the cells and stay in the G2 phase.), then transfer to galactose medium for 4 hours, $10^{8}$ cells were taken. Dilute the yeast solution to $10^{7}$ cells $/ \mathrm{mL}, 10^{6}$ cells $/ \mathrm{mL}, 10^{5}$ cells $/ \mathrm{mL}, 10^{4}$ cells $/ \mathrm{mL}$, and $10^{3}$ cells $/ \mathrm{mL}$ in pure water; inoculate a suitable amount of the diluted bacterial solution in the medium plate for about 48 hours. Observe the colony growth status, take a photo record, and save the results.

\subsection{Test of yeast survival rate}

Adequate yeast cells were transferred to a $10 \mathrm{ml}$ YPDA liquid medium in a shake flask the night before, and cultured overnight at $30^{\circ} \mathrm{C}$ shaker. After the next day of Nocodazole treatment for 3 hours (Synchronize the cells and stay in the G2 phase.), $10^{3}$ cells were taken after 4 hours of transfer to galactose medium. The cells were dissolved in 200ul sterile water and evenly coated on the galactose plate, and single colonies were counted 48 hours later.

\section{Results}

\subsection{A schematic diagram of the principle of gene rating strains that produce adjacent DSBs.}

In order to simultaneously generate two adjacent DSBs and compare the differences with one DSB, we constructed three strains. The schematic diagram of two$D S B u$ is shown in the figure1. The other two strains are $n o-D S B u$ and one-DSBu . no-DSBu has no I-SceI site, and one-DSBu has only one I-SceI site in front of the galactose promoter.

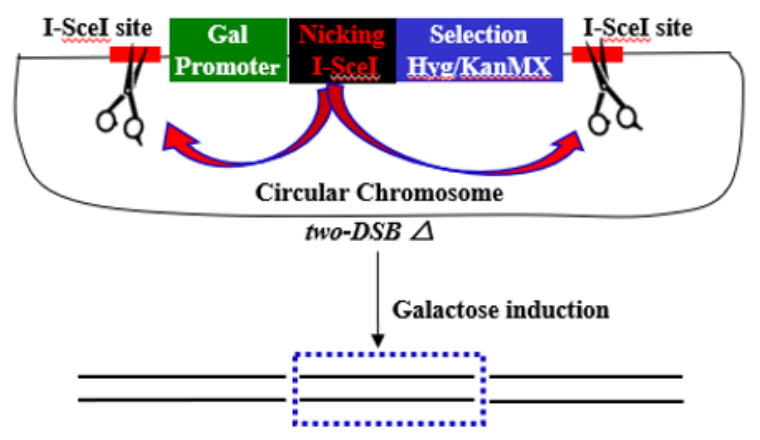

Fig. 1. Schematic diagram of constructing two adjacent DSBs strains.

\subsection{Deletion of the Rad51 gene leads to enhanced sensitivity of two adjacent DSBs strain under galactose induction.}

Sensitivity experiments to test changes in sensitivity of mutants presented in Figure 2a. Through sensitivity experiments we found that the production of a DSB in yeast cells is the same as the sensitivity of producing two DSBs. However, when the Rad50 gene and the Rad51 gene were knocked out in one-DSBu and two-
$D S B u$, the sensitivity of the $t w o-D S B u$ in which the Rad51 gene was deleted was significantly enhanced. To further verify the survival activity of the two-DSB knockout Rad51, the survival rate of multiple mutants was further examined by spore counting assay. The result is shown in Figure $2 b$. The survival rate of the strains producing DSBs decreased, but the survival rate of two$D S B$ u after the Rad51 gene was knocked out was the most obvious.

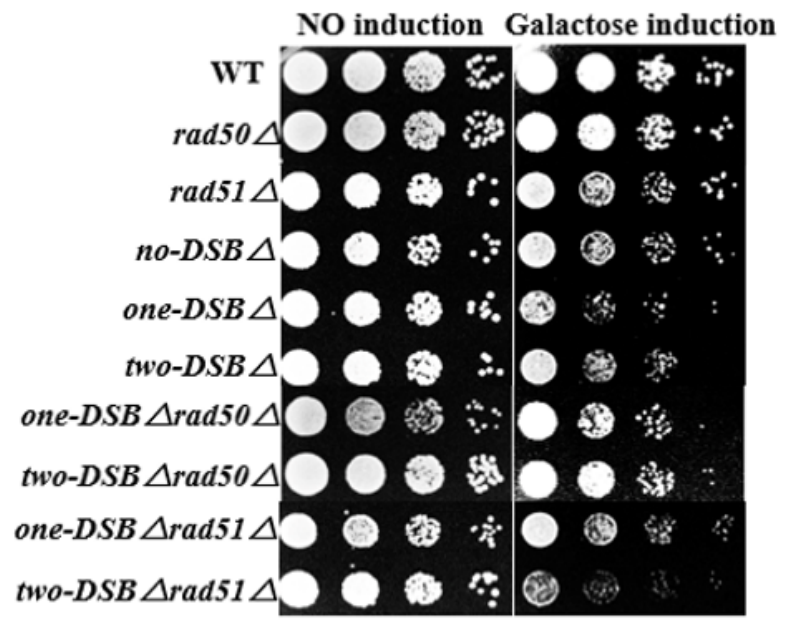

Fig. 2a. Sensitivity assay for detecting multiple mutants

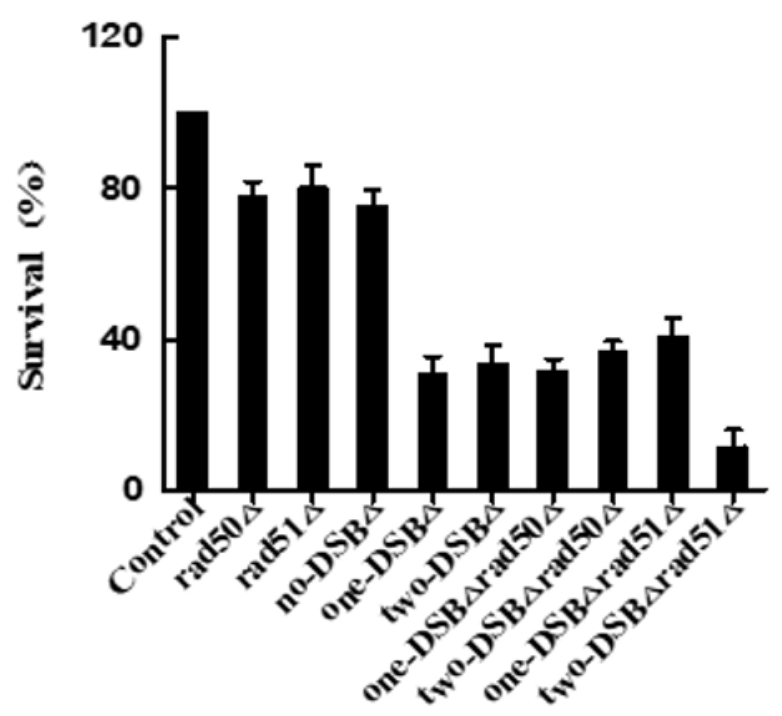

Fig. 2b. The survival rate of different mutants.

\subsection{Westhernblot test the expression of I-Scel protein.}

Westhernblot detected the expression of I-SceI protein in three mutants. as shown in the figure 3. Only by induction of galactose, no- $D S B u$, one-DSBu and two$D S B u$ could discover the expression of I-SceI protein, which proved that the production of DSBs was induced by galactose. 


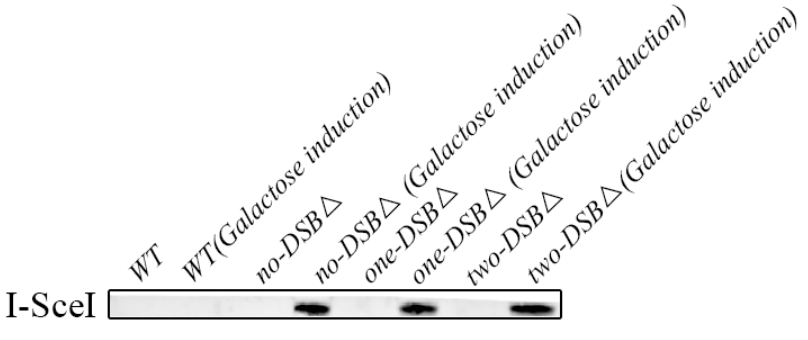

Fig. 3. Westhernblot detected the expression of I-SceI protein.

\subsection{Knock out the Rad51 gene, the effect of the distance between two adjacent DSBs on strain sensitivity.}

Schematic diagram of different cutting site distance strain construction illustrated in Figure 4a. On the basis of the no-DSB strain, a sequence with URA3 as a selection marker was inserted after the Gal-I-SceI cassette, and the I-SceI recognition site was ligated at both ends of the sequence. The recognition distance between the two ends was 1000 bps (1000two-DSB $u$ )or 4000 bps (4000two-DSB $u$ ). As shown in Figure 4b. The strain lacking Rad51 was less sensitive as the distance between the two DSBs became shorter.

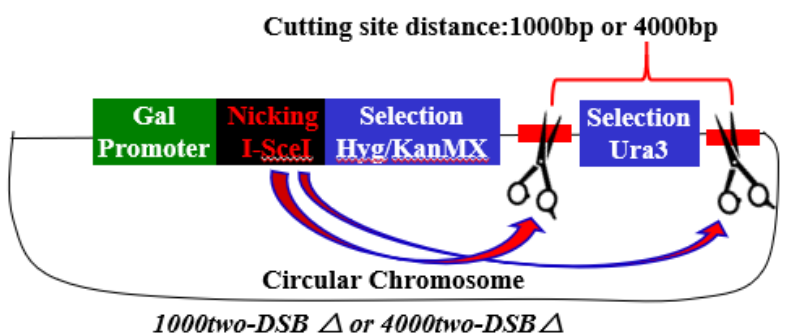

Fig. 4a. Schematic diagram of different cutting site distance strain construction.

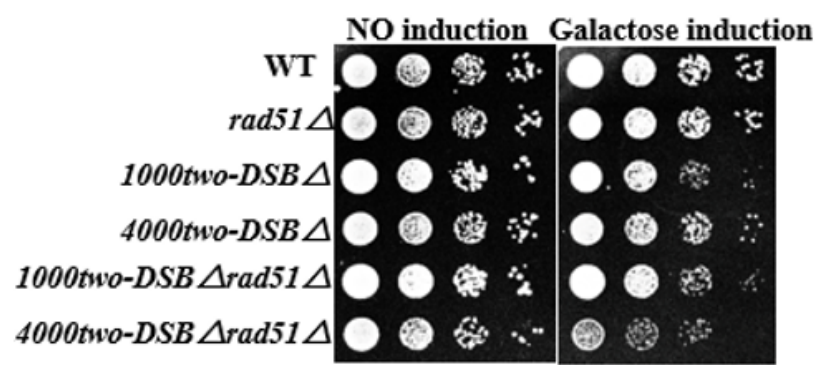

Fig. 4b. Sensitivity assay for multiple mutants.

\subsection{Explanation of the RAD51 gene deletion leading to enhanced sensitivity of the two-DSB strain.}

The deletion of Rad51 resulted in an increase in the sensitivity of two-DSBu, and the sensitivity of one-DSB did not change much. We believe that Rad51 results in stabilizing the two DNA ends. The exact reason is as shown in the figure5. When two adjacent DSBs are generated synchronously on one chromosome, if the protection of Rad51 is lost, the sequence between the two DSBs will fall off to produce a sizable gap.

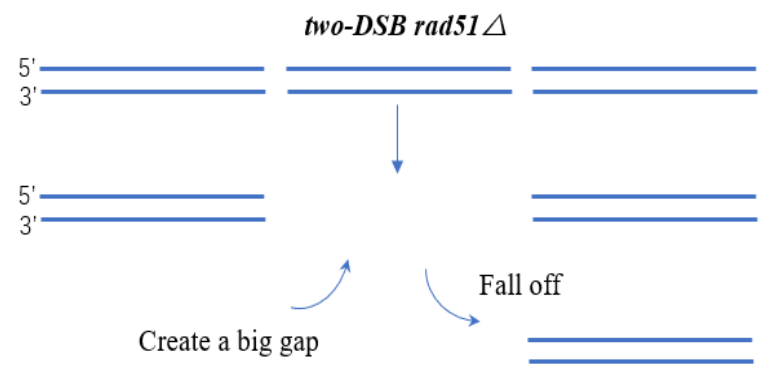

Fig. 5. Schematic diagram of deleting the RAD51 gene causes the sequence the two adjacent DSBs to fall.

\section{Conclusions}

We successfully constructed a strain that simultaneously induced the production of two adjacent DSBs on a single chromosome. And compare the difference in the repair of a DSB and two adjacent DSBs.

These data indicate that the cell viability is decreased by synchronous induction of one DSB or two adjacent DSBs on a single chromosome of yeast. After further knocking out the Rad51 gene, the viability of the strain producing two adjacent DSBs is further deepened. Subsequent experiments demonstrated that $\operatorname{Rad} 51$ has a robust effect on the sequence between two adjacent DSBs. Losing the protection of Rad51, the sequence between two adjacent DSBs drops directly, producing a huge gap.

In summary, the role of Rad51 in two adjacent DSBs allows us to further understand the mechanism of recombinant repair, even develop more functional mechanisms of Rad51. This is important to maintaining genomic stability and developing new drugs that target cancer treatment.

\section{Conflict of Interest}

We have no conflicts of interest to disclose.

\section{References}

1. L. Campos-Doerfler, S. Syed, K. H. Schmidt, Sgs1 Binding to Rad51 Stimulates Homology-Directed DNA Repair in Saccharomyces cerevisiae. Genetics 208, 125-138 (2018).

2. W. Zhao, J. B. Steinfeld, F. Liang, X. Chen, D. G. Maranon, C. Jian Ma, Y. Kwon, T. Rao, W. Wang, C. Sheng, X. Song, Y. Deng, J. Jimenez-Sainz, L. Lu, R. B. Jensen, Y. Xiong, G. M. Kupfer, C. Wiese, E. C. Greene, P. Sung, BRCA1-BARD1 promotes RAD51-mediated homologous DNA pairing. Nature 550, 360-365 (2017).

3. K. Seeliger, S. Dukowic-Schulze, R. WurzWildersinn, M. Pacher, H. Puchta, BRCA2 is a mediator of RAD51- and DMC1-facilitated 
homologous recombination in Arabidopsis thaliana. The New phytologist 193, 364-375 (2012).

4. S. Paulikova, M. Chmelarova, J. Petera, V. Palicka, A. Paulik, Hypermethylation of RAD51L3 and $\mathrm{XRCC} 2$ genes to predict late toxicity in chemoradiotherapy-treated cervical cancer patients. Folia biologica 59, 240-245 (2013).

5. T. Takenaka, I. Yoshino, H. Kouso, T. Ohba, T. Yohena, A. Osoegawa, F. Shoji, Y. Maehara, Combined evaluation of Rad51 and ERCC1 expressions for sensitivity to platinum agents in non-small cell lung cancer. International journal of cancer 121, 895-900 (2007).

6. A. P. Wiegmans, F. Al-Ejeh, N. Chee, P. Y. Yap, J. J. Gorski, L. Da Silva, E. Bolderson, G. ChenevixTrench, R. Anderson, P. T. Simpson, S. R. Lakhani, K. K. Khanna, Rad51 supports triple negative breast cancer metastasis. Oncotarget 5, 3261-3272 (2014).

7. G. Liu, D. Yang, R. Rupaimoole, C. V. Pecot, Y. Sun, L. S. Mangala, X. Li, P. Ji, D. Cogdell, L. Hu, Y. Wang, C. Rodriguez-Aguayo, G. LopezBerestein, I. Shmulevich, L. De Cecco, K. Chen, D. Mezzanzanica, F. Xue, A. K. Sood, W. Zhang, Augmentation of response to chemotherapy by microRNA-506 through regulation of RAD51 in serous ovarian cancers. Journal of the National Cancer Institute 107, (2015).

8. H. Maacke, K. Jost, S. Opitz, S. Miska, Y. Yuan, L. Hasselbach, J. Luttges, H. Kalthoff, H. W. Sturzbecher, DNA repair and recombination factor Rad51 is over-expressed in human pancreatic adenocarcinoma. Oncogene 19, 2791-2795 (2000).

9. W. Ma, M. A. Resnick, D. A. Gordenin, Apn1 and Apn2 endonucleases prevent accumulation of repairassociated DNA breaks in budding yeast as revealed by direct chromosomal analysis. Nucleic acids research 36, 1836-1846 (2008).

10. A. L. Goldstein, J. H. McCusker, Three new dominant drug resistance cassettes for gene disruption in Saccharomyces cerevisiae. Yeast (Chichester, England) 15, 1541-1553 (1999). 